\title{
Standard Heterosis of Hybrids Maize (Zea Mays L.) for Grain Yield and Yield Related Traits at Kulumsa, Southeastern Ethiopia
}

\author{
Abiy Balcha Gebre ${ }^{1 *}$, Hussein Mohammed ${ }^{2}$, Demissew Abakemal ${ }^{3}$ \\ ${ }^{1}$ Ethiopian institute of Agricultural Research, Kulumsa Agricultural Research Center, P.O.Box 489, Kulumsa, \\ Ethiopia \\ ${ }^{2}$ School of Plant and Horticultural Science. Hawassa University, P.O.Box 05, Hawassa, Ethiopia \\ ${ }^{3}$ Ethiopian institute of Agricultural Research, Ambo Agricultural Research Center, P.O.Box 37, Ambo, Ethiopia \\ *Corresponding Author: Abiy Balcha Gebre, Ethiopian institute of Agricultural Research, Kulumsa \\ Agricultural Research Center, P.O.Box 489, Kulumsa, Ethiopia
}

\begin{abstract}
Maize (Zea mays L.) is one of the most important cereal crops cultivated globally leading total yield productions of crops. The development of inbred lines and hybrids is one of the widely used breeding strategies in maize. The national average maize yield in Ethiopia is low and thus, choice of promising germplasm, knowledge of combining ability and heterosis are a prerequisite to develop high yielding maize varieties. A total of 44 maize hybrids along with two popular standard checks (Jibat and Wenchi) were evaluated in randomization complete block design replicated twice. Forty-two hybrids with 2 checks were tested to estimate standard heterosis for yield related traits during 2017 cropping season at Kulumsa Agricultural Research Center. There was significant difference between the genotypes in Grain yield (GY), Days to anthesis (DTA), Days to silking (DTS), plant height $(P H)$ ear height (EH), ear per plant (EPP), ear aspect (EA), biomass yield (BY), harvest index (HI), 1000 kernel weight (TKWT), number of kernels per row $(N K R)$, ear length (EL) and ear diameter (ED). The result of heterosis estimation showed considerable amount of positive and negative heterosis for all studied traits. The highest significant positive standard heterosis for grain yield was manifested by L6XT2 (44.54 and 48.41\%) over Jibat and Wenchi respectively. Thus, hybrid L6XT2 was the best combination in mean grain yield and standard heterosis. Hybrids L6XT2, L8XT2 and L3XT2 had higher magnitude of heterosis than checks thus, these hybrids exploited for future use and additional effort is required for the development desirable maize variety.
\end{abstract}

Keywords: Heterosis, Hybrid, Line x Tester and Yield

\section{INTRODUCTION}

Maize (Zea mays L.; $2 \mathrm{n}=20$ ) is an important cereal crop of the world, belonging to the tribe Maydeae of the grass family Poaceae. It has become a leading agricultural crop and is used for food, feed, fuel and fiber in many parts of the world, in temperate, tropical and sub-tropical regions. Wheat, rice and Maize together make-up three-fourths of the world grain production. Maize is the world's leading crop with more than 600 million tons of grains from about 138 million hectares (Ram, 2011).

In Ethiopia, the highland agro-ecology was previously known for its highland crops production including barley, wheat, broad beans, field peas and potatoes whereby maize was a homestead crop of minor importance (Demissew et al., 2013). This agro-ecology is densely populated and limited lands are available for crop production. Development of nutritionally enhanced and high yielding maize cultivars in this environment would enhance food and nutritional security. Besides, introduction of improved highland maize genotypes may diversify the cropping system and significantly improve the livelihoods of poor households. Recent research developments for highland maize improvement program in Ethiopia hold out the hope for attaining this goal.

The term heterosis was coined by Shull (1952). He defined heterosis as increased vigor, size, fruitfulness, speed of development, resistance to disease and pest, or to climatic changes of any kind, manifested by cross bred organisms as compared with corresponding inbred. Heterosis is usually described in terms of the superiority of F1 hybrid performance over some measure of parental 
performance; that means definition of heterosis differs depending on the basis of comparison used. Accordingly, heterosis is measured as improvement of F1 over the mean of both parents (mid parent heterosis or relative heterosis) (Falconer and Mackey, 1996; Stuber, 1999); over the mean of the better parent or hetrobeltiosis (Surendran et al., 1994).

Heterosis was first exploited in the 1930s with large-scale production of hybrid maize. This commercial success of hybrid maize prompted efforts to commercialize hybridization in cross and self-pollinated field and vegetable crops (Hunter, 1999; Duvic, 1999). Commercialization of heterosis has also been one of the driving forces in the rapid development of privately and public funded breeding research programs around the world, which in turn have provided good opportunities for the researchers to further advance their knowledge in this field. Indeed, hybrid varieties have contributed greatly worldwide to the production of many crop species (Stuber, 1994). From a commercial point of view, however heterosis may also be measured as the degree of hybrid performance over the best available variety and this is called standard heterosis (Virmani and Edwards, 1983)

Thus, the present study was conducted to determine the magnitude of standard heterosis for yield and yield related traits and to identify potential hybrids for future breeding schemes.

\section{MATERial AND Method}

\subsection{Description of the Study Area}

The experiment was conducted at Kulumsa agricultural research center, during the main cropping season of 2018. It lies at $8^{\circ} 5^{\prime} \mathrm{N}$ latitude, $39^{\circ} 10^{\prime} \mathrm{E}$ longitude with an altitude of 2200 m.a.s.l and is located in a tepid to cool, moist plain agro-ecological zone. Rainfall data for the last 10 years (20062016) showed that average total annual rainfall was $830 \mathrm{~mm}$ which ranged from 535 to $1018 \mathrm{~mm}$. Average annual maximum temperature was $23.2{ }^{\circ} \mathrm{C}$ and minimum temperature was $10{ }^{\circ} \mathrm{C}$. The dominant soil type is luvisol/eutric nitosols with good drainage and $(\mathrm{pH}=6)$.

\subsection{Experimental Material, Design and Management}

The material comprised of 21 early generation elite inbred lines coded as L1, L2, L3...L21 and two testers coded as $\mathrm{T} 1$ and $\mathrm{T} 2$. The inbred lines crossed with tester in line $\mathrm{x}$ tester mating design to generate 42 F1 hybrids at Ambo Agricultural Research Center in cropping seasons (2016). The crosses were planted along with two commercial checks (Jibat and Wenchi) to made available to evaluate in a randomized complete block design with two replications of one row plot in 2017 rainy season. The testers are line testers and checks are three-way cross of normal hybrid maize. Both are used in this study are locally developed by the national highland maize breeding program in Ethiopia.

Each plot had one row of 5.25 meter length ( 21 plants). Each genotype was planted at $75 \mathrm{~cm}$ between rows and $25 \mathrm{~cm}$ within row spacing. Two seeds were planted per hill on $23^{\text {th }}$ of April and later thinned to one plant per hill providing a uniform stand of about 53,333 plants ha ${ }^{-1}$.

All cultural practices like weeding and cultivation was done manually throughout the entire growing season as required. Data of 12 quantitative traits were collected viz., days to $50 \%$ emergence, days to $50 \%$ anthesis, days to $50 \%$ silking, days to maturity, plant height, ear height, ear length, number of rows per ear, ear diameter and number of kernels per row, 1000 kernel weight and grain yield later converted to mean values.

\subsection{Statistical Analysis}

Data were subjected to analyses of variance using PROC GLM procedure in SAS software version 9.2 (SAS, 2010). In the analysis, entries were used as fixed factor while replications were considered as random factors. This was specified using RANDOM statement in PROC GLM model of SAS. The magnitude of heterosis was estimated in relation to standard checks for traits that showed significant differences following the method suggested by Falconer and Mackay (1996).

$\mathrm{SH} \%=\mathrm{F} 1-\mathrm{SV} \quad \mathrm{X} 100$

$$
\text { SV }
$$

$\mathrm{F} 1=$ Mean value of a cross

$\mathrm{SV}=$ Mean value of standard check

$\mathrm{SH}=$ Standard heterosis 
The differences in the magnitude of heterosis tested following the procedure of Panse V.G. and P.V. Sukhatme, (1961). T-test was used for significance test among crosses and checks. Standard error and critical difference were computed as:

$\mathrm{SE}(\mathrm{d})=(2 \mathrm{Me} / \mathrm{r})^{1 / 2}$

$\mathrm{CD}=\mathrm{SE}(\mathrm{d}) \times \mathrm{t}$

$\mathrm{t}=$ value of $\mathrm{t}$ at error degree of freedom

The computed t-value was tested against the t-value at error degree of freedom corresponding to 5 or $1 \%$ level of significance.

\section{RESUltS}

Analysis of variance showed that mean squares of genotype were significant $(\mathrm{P}<0.01)$ for traits such as grain yield, days to anthesis and silking, ear height, number of ears per plant, ear aspect, biomass yield, harvest index, ear length and ear diameter and significant $(\mathrm{P}<0.05)$ for traits like plant height, thousand kernel weight and number of kernels per row. Non-significant differences were obtained for anthesis-silking interval, days to maturity, plant aspect and number of rows per ear (Table 1).

Table1. Mean squares for grain yield and yield related traits in 42 test crosses and 2 checks evaluated at Kulumsa, 2017

\begin{tabular}{|c|c|c|c|c|c|c|c|c|c|}
\hline $\begin{array}{l}\text { Source of } \\
\text { variation }\end{array}$ & df. & $\begin{array}{c}\text { GY } \\
\text { (t/ha) }\end{array}$ & $\begin{array}{c}\text { DTA } \\
\text { (days) }\end{array}$ & $\begin{array}{c}\text { DTS } \\
\text { (days) }\end{array}$ & $\begin{array}{c}\text { ASI } \\
\text { (days) }\end{array}$ & $\begin{array}{c}\text { DTM } \\
(\text { days })\end{array}$ & $\begin{array}{c}\text { PH } \\
(\mathrm{cm})\end{array}$ & $\begin{array}{c}\text { EH } \\
(\mathrm{cm})\end{array}$ & $\begin{array}{c}\text { EPP } \\
(\#)\end{array}$ \\
\hline Replication & 1 & 0.38 & 0.01 & 0.91 & 0.1 & 6.3 & 823.44 & 278.68 & 0.07 \\
\hline Genotype(G) & 43 & $4.59^{* *}$ & $23.27^{* *}$ & $21.65^{* *}$ & 0.9 & 8.66 & $289.46^{*}$ & $321.93^{* *}$ & $0.12^{* *}$ \\
\hline Crosses (Cr) & 41 & $4.39 * *$ & $21.13 * *$ & $19.07 * *$ & 0.87 & 8.18 & $246.06^{*}$ & $291.89 * *$ & $0.12^{* *}$ \\
\hline Error & 43 & 1.39 & 6.53 & 4.71 & 0.89 & 11.64 & 144.25 & 118.90 & 0.03 \\
\hline CV $(\%)$ & - & 13.21 & 3.13 & 2.57 & 33.13 & 1.85 & 4.95 & 7.72 & 12.41 \\
\hline
\end{tabular}

Note: $*, * *=$ Significant at 0.05 and 0.01 levels of probability, respectively, ASI=anthesis silking interval, DTA= days to anthesis, DTM=days to maturity, DTS= days to silking, EH=ear height, EPP =number of ears per plant, $G Y=$ grain yield, $P H=$ plant height

Table1. Continued

\begin{tabular}{|c|c|c|c|c|c|c|c|c|c|c|}
\hline $\begin{array}{c}\text { Source of } \\
\text { variation }\end{array}$ & df. & $\begin{array}{c}\text { EA } \\
(\#)\end{array}$ & $\begin{array}{c}\text { PA } \\
(\#)\end{array}$ & $\begin{array}{c}\text { BY } \\
(\mathrm{t} / \mathrm{ha})\end{array}$ & $\begin{array}{c}\text { HI } \\
(\%)\end{array}$ & $\begin{array}{c}\text { TKWT } \\
(\mathrm{g})\end{array}$ & $\begin{array}{c}\text { NKR } \\
(\#)\end{array}$ & $\begin{array}{c}\text { RPE } \\
(\#)\end{array}$ & $\begin{array}{c}\text { EL } \\
(\mathrm{cm})\end{array}$ & $\begin{array}{c}\text { ED } \\
(\mathrm{cm})\end{array}$ \\
\hline Replication & 1 & 0.24 & 0 & 0.05 & 14.62 & 4367.53 & 46.21 & 0.58 & 0.15 & 0.03 \\
\hline Genotype(G) & 43 & $0.37^{* *}$ & 0.15 & $27.70^{* *}$ & $138.88^{* *}$ & $3995.21^{*}$ & $17.68^{*}$ & 0.68 & $1.31^{* *}$ & $0.12^{* *}$ \\
\hline Cross (Cr) & 41 & $0.35^{* *}$ & 0.15 & $24.98^{* *}$ & $144.49^{* *}$ & $4111.62^{*}$ & 17.38 & 0.64 & $1.25^{* *}$ & $0.13^{* *}$ \\
\hline Error & 43 & 0.16 & 0.17 & 3.02 & 41.71 & 2608.82 & 10.15 & 1.11 & 0.31 & 0.15 \\
\hline CV (\%) & - & 16.18 & 17.34 & 8.69 & 11.45 & 12.33 & 8.91 & 8.91 & 3.08 & 2.77 \\
\hline
\end{tabular}

Note: *, **, = Significant at 0.05 and 0.01 levels of probability, respectively, BY= biomass yield, EA=ear aspect, $E D=$ ear diameter, $E L=$ ear length, $H I=$ harvest index, $N K R=$ number of kernels per row, PA=plant aspect, $R P E=$ Number of rows per ear, TKWT=thousand kernels weight

The estimates of heterosis over the best standard check were computed for grain yield and yield related traits that showed significant differences among genotypes. Both standard checks used to estimate standard heterosis produce below mean grain yield per hectare. Jibat was relativity the best standard check in mean grain yield per hectare than Wenchi. Significant amount of heterosis was observed for all the traits under study; however, the magnitude varied with traits (Table 3)

Grain Yield: Among the 42 crosses, 7 crosses exhibited positive and significant to highly significant heterosis over the best standard check (Jibat) for grain yield (Table 3). Standard heterosis for this trait ranged from $44.54(\mathrm{~L} 6 \times \mathrm{T} 2)$ to $-28.66 \%$ (L3 x T1). L6 x T2 (44.54 \%), L8 x T2 (42.93\%), L3 x T2 $(42.43 \%)$ exhibited higher standard heterosis over Jibat. Similarly, the standard heterosis over Wenchi varied from 48.11 (L6 x T2) to $-26.75 \%$ (L3 x T1) and L6 x T2 (48.11\%), L8 x T2 (48.75 $\%)$, L3 x T2 (46.24 \%) exhibited positive higher standard heterosis (Table 4). Positive heterosis is desirable as it indicates increased yield over the existing standard check. In agreement with the current finding, the expression of grain yield heterosis above the standard check in maize has been reported by several investigators (Gupta et al., 1994; Vengopal et al., 2002; Tiwari, 2003; Twumasi et al., 2003; Amiruzzaman et al., 2010; Wali et al., 2010). 
Standard Heterosis of Hybrids Maize (Zea Mays L.) for Grain Yield and Yield Related Traits at Kulumsa, Southeastern Ethiopia

Table3. The magnitude of standard heterosis for 42 hybrids over Jibat and Wenchi for grain yield and related traits evaluated at Kulumsa, 2017.

\begin{tabular}{|c|c|c|c|c|c|c|c|c|c|c|}
\hline \multirow[b]{2}{*}{ Crosses } & \multicolumn{2}{|c|}{ GY } & \multicolumn{2}{|r|}{ DTA } & \multicolumn{2}{|r|}{ DTS } & \multicolumn{2}{|c|}{$\mathrm{PH}$} & \multicolumn{2}{|c|}{$\mathrm{EH}$} \\
\hline & Jibat & Wenchi & Jibat & Wenchi & Jibat & Wenchi & Jibat & Wenchi & Jibat & Wenchi \\
\hline L1 x T1 & $33.62 *$ & $37.20 *$ & -3.91 & -1.15 & -4.32 & -2.75 & $18.32 * *$ & $30.40 * *$ & $34.64 * *$ & $69.82 * *$ \\
\hline L1 x T2 & -6.70 & -4.20 & $-12.29 * *$ & $-9.77 * *$ & $-12.43 * *$ & $-10.99 * *$ & -0.86 & 9.26 & -12.86 & 9.91 \\
\hline $\mathrm{L} 2 \times \mathrm{T} 1$ & 20.47 & 23.69 & 0.00 & 2.87 & 0.00 & 1.65 & 6.90 & $17.81 * *$ & -2.50 & $22.97 *$ \\
\hline L2 x T2 & 23.45 & 26.75 & -4.47 & -1.72 & $-5.95 * *$ & -4.40 & $11.21 *$ & $22.57 * *$ & 2.86 & $29.73^{* *}$ \\
\hline L3 x T1 & -28.66 & -26.75 & -7.82 & $-5.17 *$ & $-7.57 * *$ & $-6.04 * *$ & 5.39 & $16.15^{* *}$ & 3.57 & $30.63^{* *}$ \\
\hline $\mathrm{L} 3 \times \mathrm{T} 2$ & $42.43 * *$ & $46.24 * *$ & -4.47 & -1.72 & -5.41 & -3.85 & 9.70 & $20.90 * *$ & 6.43 & $34.23 * *$ \\
\hline L4 x T1 & 7.32 & 10.19 & $-10.06^{* *}$ & $-7.47 * *$ & $-9.73 * *$ & $-8.24 * *$ & 5.82 & $16.63 * *$ & 3.21 & $30.18^{* *}$ \\
\hline $\mathrm{L} 4 \times \mathrm{T} 2$ & $30.89 *$ & 34.39 & -4.47 & -1.72 & -4.86 & -3.30 & 6.47 & $17.34 * *$ & 10.71 & $39.64 * *$ \\
\hline L5 x T1 & -11.66 & -9.30 & $-10.06^{* *}$ & $-7.47 * *$ & $-9.73 * *$ & $-8.24 * *$ & 3.88 & $14.49 * *$ & 7.14 & $35.14 * *$ \\
\hline L5 x T2 & 24.81 & 28.15 & -8.38 & $-5.75 *$ & $-8.11 * *$ & $-6.59 * *$ & 5.39 & $16.15^{* *}$ & 7.14 & $35.14 * *$ \\
\hline L6 x T1 & 1.49 & 4.20 & $-15.64 * *$ & $-13.22 * *$ & $-15.14 * *$ & $-13.74 * *$ & $11.42 *$ & $22.80 * *$ & $15.71 *$ & $45.95 * *$ \\
\hline L6 x T2 & $44.54 * *$ & $48.41 * *$ & $-5.59 *$ & -2.87 & -5.41 & -3.85 & 8.84 & $19.95 * *$ & 8.93 & $37.39 * *$ \\
\hline L7 x T1 & -13.77 & -11.46 & $-9.50 * *$ & $-6.90 * *$ & $-9.19 * *$ & $-7.69 * *$ & 9.48 & $20.67 * *$ & 9.64 & $38.29 * *$ \\
\hline $\mathrm{L} 7 \times \mathrm{T} 2$ & 22.21 & 25.48 & $-6.70 * *$ & -4.02 & $-7.03 * *$ & $-5.49 *$ & 1.72 & $12.11^{*}$ & 6.07 & $33.78 * *$ \\
\hline L8 x T1 & -13.15 & -10.83 & $-12.85 * *$ & $-10.34 * *$ & $-12.97 * *$ & $-11.54 * *$ & -1.94 & 8.08 & -3.57 & $21.62 *$ \\
\hline $\mathrm{L} 8 \times \mathrm{T} 2$ & $42.93 * *$ & $46.75 * *$ & $-10.61 * *$ & $-8.05 * *$ & $-10.27 * *$ & $-8.79 * *$ & 6.90 & $17.81 * *$ & 10.71 & $39.64 * *$ \\
\hline L9 x T1 & -2.36 & 0.25 & $-11.73 * *$ & $-9.20 * *$ & $-11.35^{* *}$ & $-9.89 * *$ & -0.22 & 9.98 & -9.29 & 14.41 \\
\hline L9 x T2 & 9.43 & 12.36 & $-5.03 *$ & -2.30 & -5.41 & -3.85 & 4.53 & $15.20 * *$ & 11.43 & $40.54 * *$ \\
\hline L10x T1 & -6.45 & -3.95 & $-10.61 * *$ & $-8.05 * *$ & $-11.35^{* *}$ & $-9.89 * *$ & 0.86 & 11.16 & -8.21 & 15.77 \\
\hline $\mathrm{L} 10 \times \mathrm{T} 2$ & $34.00 *$ & $37.58 *$ & $-8.38 * *$ & $-5.75^{*}$ & $-7.57 * *$ & $-6.04 * *$ & 7.33 & $18.29 * *$ & 2.14 & $28.83^{* *}$ \\
\hline $\mathrm{L} 11 \times \mathrm{T} 1$ & -4.96 & -2.42 & $-12.29 * *$ & $-9.77 * *$ & $-11.89 * *$ & $-10.44 * *$ & 7.97 & $19.00 * *$ & 0.36 & $26.58 * *$ \\
\hline $\mathrm{L} 11 \times \mathrm{T} 2$ & 19.60 & 22.80 & $-11.73 * *$ & $-9.20 * *$ & $-11.35^{* *}$ & $-9.89 * *$ & 3.23 & $13.78^{*}$ & 0.71 & $27.03^{* *}$ \\
\hline L12 x T1 & -4.34 & -1.78 & $-14.53 * *$ & $-12.07 * *$ & $-14.59 * *$ & $-13.19 * *$ & 3.23 & $13.78^{*}$ & -0.71 & $25.23 *$ \\
\hline L12 x T2 & 29.16 & $32.61 *$ & $-10.06^{* *}$ & $-7.47 * *$ & $-8.65 * *$ & $-7.14 * *$ & 6.68 & $17.58^{* *}$ & 4.64 & $31.98 * *$ \\
\hline L13x T1 & -11.91 & -9.55 & $-6.70 * *$ & -4.02 & $-7.03 * *$ & $-5.49 *$ & 5.17 & $15.91 * *$ & 7.14 & $35.14 * *$ \\
\hline L13x T2 & 27.67 & $31.08 *$ & $-7.82 * *$ & $-5.17 *$ & $-7.57 * *$ & $-6.04 * *$ & 4.31 & 14.96 & -5.36 & 19.37 \\
\hline L14 x T1 & -3.35 & -0.76 & $-11.73 * *$ & $-9.20 * *$ & $-11.35^{* *}$ & $-9.89 * *$ & -1.08 & 9.03 & -4.29 & $20.72 *$ \\
\hline $\mathrm{L} 14 \times \mathrm{T} 2$ & 14.39 & 17.45 & $-13.41 * *$ & $-10.92 * *$ & $-10.27 * *$ & $-8.79 * *$ & $12.28 *$ & $23.75^{* *}$ & -1.43 & $24.32 *$ \\
\hline $\mathrm{L} 15 \times \mathrm{T} 1$ & 4.71 & & $-12.29 * *$ & $-9.77 * *$ & $-11.35^{* *}$ & $-9.89 * *$ & 2.16 & $12.59 *$ & -3.21 & $22.07 *$ \\
\hline $\mathrm{L} 15 \times \mathrm{T} 2$ & 11.04 & 14.01 & $-12.29 * *$ & $-9.77 * *$ & $-12.43^{* *}$ & $-10.99 * *$ & 5.17 & $15.91 * *$ & -2.50 & $22.97 * *$ \\
\hline L16x T1 & 4.22 & & $-12.29 * *$ & $-9.77 * *$ & $-12.97 * *$ & $-11.54 * *$ & 6.03 & $16.86^{* *}$ & -7.50 & 16.67 \\
\hline $\mathrm{L} 16 \times \mathrm{T} 2$ & 28.91 & $32.36^{*}$ & $-5.59 *$ & -2.87 & $-5.95 *$ & -4.40 & 3.66 & $14.25 *$ & -0.36 & $25.68 *$ \\
\hline $\mathrm{L} 17 \times \mathrm{T} 1$ & -10.79 & -8.41 & $-11.73 * *$ & $-9.20 * *$ & $-10.81 * *$ & $-9.34 * *$ & 5.82 & $16.63 * *$ & 0.71 & $27.03 * *$ \\
\hline $\mathrm{L} 17 \times \mathrm{T} 2$ & 16.50 & 19.62 & $-8.94 * *$ & $-6.32 * *$ & $-9.19 * *$ & $-7.69 * *$ & 5.17 & $15.91 * *$ & 3.21 & $30.18 * *$ \\
\hline L18 x T1 & 7.07 & 9.94 & $-12.85 * *$ & $-10.34 * *$ & $-12.97 * *$ & $-11.54 * *$ & $-10.78 *$ & -1.66 & -10.00 & 13.51 \\
\hline $\mathrm{L} 18 \times \mathrm{T} 2$ & $31.39 *$ & $34.90 *$ & $-10.06 * *$ & $-7.47 * *$ & $-10.27 * *$ & $-8.79 * *$ & 6.47 & $17.34 * *$ & -0.71 & $25.23 *$ \\
\hline L19 x T1 & -2.36 & 0.25 & $-12.29 * *$ & $-9.77 * *$ & $-11.35^{* *}$ & $-9.89 * *$ & 8.19 & $19.24 * *$ & -7.50 & 16.67 \\
\hline $\mathrm{L} 19 \times \mathrm{T} 2$ & 2.48 & 5.22 & -3.91 & -1.15 & -5.41 & -3.85 & 3.02 & $13.54 *$ & -0.36 & $25.68 *$ \\
\hline $\mathrm{L} 20 \times \mathrm{T} 1$ & -11.66 & -9.30 & $-9.50 * *$ & $-6.90 * *$ & $-9.19 * *$ & $-7.69 * *$ & 0.65 & 10.93 & -10.36 & 13.06 \\
\hline $\mathrm{L} 20 \times \mathrm{T} 2$ & 6.95 & 9.81 & -2.79 & 0.00 & -2.70 & -1.10 & 7.54 & $18.53 * *$ & 5.71 & $33.33 * *$ \\
\hline $\mathrm{L} 21 \times \mathrm{T} 1$ & 0.25 & 2.93 & $-12.29 * *$ & $-9.77 * *$ & $-11.89 * *$ & $-10.44^{* *}$ & 5.39 & $16.15^{* *}$ & -4.29 & $20.72 *$ \\
\hline $\mathrm{L} 21 \times \mathrm{T} 2$ & -6.45 & -3.95 & $-5.03 *$ & -2.30 & -5.41 & -3.85 & -2.59 & 7.36 & -8.93 & 14.86 \\
\hline $\mathrm{SE}( \pm)$ & 0.83 & & 1.53 & & 1.81 & & 8.49 & & 7.71 & \\
\hline CD 0.05 & 2.38 & & 4.38 & & 5.15 & & 24.22 & & 21.99 & \\
\hline CD 0.01 & 3.18 & & 5.85 & & 6.89 & & 32.37 & & 29.39 & \\
\hline
\end{tabular}

Note: $S E(m \pm)=$ standard error of a mean, $C D=$ critical difference.

Plant Height: Negative heterosis for plant height is desirable for breeding short statured hybrids and which implying that these hybrids would mature earlier. Percent of standard heterosis over the two standard checks Jibat and Wenchi for plant height ranged from -10.78 to $18.32 \mathrm{~cm}$ and -1.66 to 30.40 $\mathrm{cm}$, respectively (Table 3 ).

Ear Height: Negative heterosis for ear height desirable implying that these hybrids could escape drought. Non hybrids showed significant negative standard heterosis for ear height over Jibat and Wenchi while two hybrids showed significant positive standard heterosis for ear height over Jibat and 
Standard Heterosis of Hybrids Maize (Zea Mays L.) for Grain Yield and Yield Related Traits at Kulumsa, Southeastern Ethiopia

twenty hybrids showed highly significant positive standard heterosis for ear height over Wenchi (Table 3).

1000 kernel weight: Standard heterosis for 1000 kernel weight varied from -13.49 (L1 x T1) to 34.02 $\%$ (L3 x T2). Positive and highly significant and significant standard heterosis manifested in ten crosses over Jibat. Although the same cross L3 x T2 $(31.10 \%)$ showed positive and highly significant heterosis over Wenchi (Table 4). Over Wenchi, eight crosses showed positive and significant heterosis ranging from -15.38 (L13 x T1) to $31.10 \%$ (L3 x T2).

Number of kernels per row: Number of kernels per row showed a standard heterosis over the Jibat varying from -0.87 (L14 x T2) to $43.46 \%$ (L12 x T1). 23 of the crosses manifested positive highly significance and significance heterosis. The result of standard heterosis computed against Wenchi showed that 11 crosses manifested positive and significant heterosis varying from -5.99 (L14 x T2) to $34.72 \%$ (L1 x T1) (Table 4). Tiwari (2003),Amiruzzama et al. (2010), Wali et al., 2010) also reported similar results in maize.

Table4. The magnitude of standard heterosis for 42 hybrids over Jibat and Wenchi for grain yield and related traits evaluated at Kulumsa, 2017.

\begin{tabular}{|c|c|c|c|c|c|c|c|c|c|c|}
\hline \multirow[b]{2}{*}{ Crosses } & \multicolumn{2}{|c|}{ BY } & \multicolumn{2}{|r|}{ TKWT } & \multicolumn{2}{|r|}{ NKR } & \multicolumn{2}{|c|}{ EL } & \multicolumn{2}{|c|}{ ED } \\
\hline & Jibat & Wenchi & Jibat & Wenchi & Jibat & Wenchi & Jibat & Wenchi & Jibat & Wenchi \\
\hline L1 x T1 & 23.15 & 26.27 & -13.49 & -15.38 & $42.06^{* * *}$ & 34.72 ** & 4.24 & 1.78 & -2.50 & -1.27 \\
\hline L1 x T2 & 20.06 & 23.10 & 13.51 & 11.03 & $30.19 * *$ & $23.47^{*}$ & 2.61 & 0.18 & 7.00 & 8.35 \\
\hline L2 x T1 & 25.93 & 29.11 & 0.04 & -2.14 & $32.29 * *$ & $25.46^{*}$ & 2.73 & 0.30 & -1.75 & -0.51 \\
\hline L2 x T2 & 19.01 & 22.03 & 17.30 & 14.74 & 13.44 & 7.58 & 5.33 & 2.84 & $10.00 *$ & $11.39 *$ \\
\hline L3 x T1 & -23.15 & -21.20 & 7.87 & 5.52 & $27.57 *$ & $20.99 *$ & $9.09 *$ & 6.51 & 6.25 & 7.59 \\
\hline L3 x T2 & 27.65 & 30.89 & $34.02 * *$ & $31.10 * *$ & 14.49 & 8.57 & 2.61 & 0.18 & 6.25 & 7.59 \\
\hline L4 x T1 & 27.65 & 30.89 & 15.56 & 13.04 & 18.32 & 12.21 & 7.88 & 5.33 & 2.00 & 3.29 \\
\hline L4 x T2 & 22.41 & 25.51 & 15.07 & 12.56 & 19.02 & 12.88 & $11.39 * *$ & $8.76^{*}$ & 17.50 ** & $18.99 *$ \\
\hline L5 x T1 & -4.75 & -2.34 & $26.75^{*}$ & $23.99 *$ & 4.54 & -0.86 & 5.33 & 2.84 & 8.25 & $9.62 *$ \\
\hline L5 x T2 & $49.88 * *$ & $53.67 * *$ & 31.48 ** & $28.62 *$ & 8.38 & 2.78 & 3.03 & 0.59 & 14.50 ** & $15.95^{* *}$ \\
\hline L6 x T1 & 29.94 & $33.23 *$ & $23.75^{*}$ & 21.06 & 7.33 & 1.79 & $\begin{array}{l}-3.03 \\
\end{array}$ & $\begin{array}{l}-5.33 \\
\end{array}$ & 2.50 & 3.80 \\
\hline L6 x T2 & $31.67 *$ & $35.00 *$ & 11.49 & 9.06 & 20.59 & 14.37 & 6.97 & 4.44 & $15.00^{* * *}$ & $16.46^{* *}$ \\
\hline L7 x T1 & 1.73 & 4.30 & 15.13 & 12.63 & $30.19^{*}$ & $23.47^{*}$ & -1.94 & -4.26 & $11.25^{*}$ & $12.66 * *$ \\
\hline L7 x T2 & $33.83 *$ & $37.22 *$ & $25.31 *$ & 22.58 & 13.44 & 7.58 & 2.42 & 0.00 & $10.00 *$ & $11.39^{*}$ \\
\hline L8 x T1 & 9.26 & 12.03 & 21.46 & 18.81 & 18.50 & 12.38 & 5.94 & 3. & 3.75 & 5.06 \\
\hline L8 x T2 & 19.01 & 22.03 & 3.54 & 1.28 & 17.28 & 11.22 & $9.70^{*}$ & 7.10 & $17.00^{* * *}$ & $18.48 * *$ \\
\hline L9 $x$ T1 & 1.11 & 3.67 & $29.87 *$ & $27.04 *$ & $24.78^{*}$ & 18.34 & -1.64 & -3.96 & 12.00 ** & $13.42 * *$ \\
\hline L9 $\times$ T2 & 25.19 & 28.35 & $25.90 *$ & $23.16^{*}$ & 10.47 & 4.77 & 5.15 & 2.66 & $13.25^{* *}$ & $14.68 * *$ \\
\hline L10x T1 & 25.31 & 28.48 & 32.39 ** & $29.51 *$ & 22.69 & 16.35 & 1.52 & -0.89 & 15.00 *** & $16.46^{* * *}$ \\
\hline L10 x T2 & 29.32 & $32.59^{*}$ & -2.60 & -4.73 & $27.05^{*}$ & $20.49^{*}$ & $10.48 *$ & 7.87 & 6.25 & 7.59 \\
\hline L11 x T1 & 24.88 & 4 & 21.92 & 19.27 & 16.23 & 10.23 & -1.64 & -3.96 & 15.00 ** & $16.46^{* * *}$ \\
\hline L11 x T2 & 22.41 & 5.51 & 11.23 & 8.81 & $25.13^{*}$ & 18.67 & $8.79 *$ & 6.21 & 3.25 & 4.56 \\
\hline L12 x T1 & $39.38^{*}$ & $42.91 * *$ & 23.38 & 20.69 & $43.46^{* *}$ & $36.05^{* *}$ & $9.09 *$ & 6.51 & $10.00^{*}$ & $11.39 *$ \\
\hline L12 x T2 & 6.05 & 8.73 & 8.52 & 6.15 & $31.94 * *$ & $25.12 *$ & 9.88* & 7.28 & 5.00 & 6.33 \\
\hline L13x T1 & -5.86 & -3.48 & 2.48 & 0.25 & $33.16^{* *}$ & $26.28^{*}$ & $8.18 *$ & 5.62 & $14.50^{* * *}$ & $15.95^{* *}$ \\
\hline L13x T2 & 24.69 & 27.85 & $29.61 *$ & $26.78^{*}$ & $23.56^{*}$ & 17.18 & $12.00 * *$ & $9.35^{*}$ & 5.00 & 6.33 \\
\hline L14 x T1 & 9.14 & 11.90 & 8.00 & 5.64 & $22.69 *$ & 16.35 & 1.21 & -1.18 & $9.50 *$ & $10.89^{*}$ \\
\hline L14 x T2 & -6.79 & -4. & .41 & 12.89 & -0.87 & -5.99 & $8.36^{*}$ & 5.80 & 3.75 & 5.06 \\
\hline L15 x T1 & -12.16 & -9.94 & 5.61 & 3.30 & 10.47 & 4.77 & -0.42 & -2.78 & $10.00 *$ & $11.39^{*}$ \\
\hline L15 x T2 & 6.98 & 9.68 & $29.72 *$ & $26.89 *$ & 17.10 & 11.06 & 4.12 & 1.66 & -1.75 & -0.51 \\
\hline L16x T1 & 11.73 & 14.56 & 19.69 & 17.08 & $22.86^{*}$ & 16.52 & 2.91 & 0.47 & $8.75 *$ & $10.13^{*}$ \\
\hline L16 x T2 & $37.35^{*}$ & $40.82^{*}$ & 11.83 & 9.39 & $22.69 *$ & 16.35 & 4.12 & 1.66 & $12.00^{* * *}$ & $13.42 * *$ \\
\hline L17 x T1 & 9.14 & 11.90 & 7.05 & 4.72 & $20.24 *$ & 14.04 & $9.39 *$ & 6.80 & $11.25^{*}$ & $12.66 * *$ \\
\hline L17 x T2 & $69.63 * *$ & 73.92** & 6.70 & 4.37 & 17.28 & 11.22 & 3.64 & 1.18 & 2.50 & 3.80 \\
\hline L18 x T1 & 18.52 & 21.52 & 14.03 & 11.55 & $25.31 *$ & 18.83 & 4.85 & 2.37 & -0.50 & 0.76 \\
\hline L18 x T2 & $56.98 * *$ & $60.95 * *$ & 18.40 & 15.82 & $21.99 *$ & 15.69 & 7.15 & 4.62 & $15.00^{* * *}$ & $16.46^{* * *}$ \\
\hline L19 x T1 & 6.79 & 9.49 & 17.51 & 14.95 & $26.88^{*}$ & 20.32 & 5.03 & 2.54 & 7.50 & $8.86^{*}$ \\
\hline L19 x T2 & 30.43 & $33.73^{*}$ & 14.55 & 12.05 & $21.82 *$ & 15.52 & 1.70 & $\begin{array}{l}-0.71 \\
\end{array}$ & $8.75^{*}$ & $10.13^{*}$ \\
\hline L20 x T1 & -4.94 & -2.53 & 14.60 & 12.10 & $35.36^{* *}$ & $28.37 * *$ & 6.67 & 4.14 & 8.25 & $9.62 *$ \\
\hline
\end{tabular}


Standard Heterosis of Hybrids Maize (Zea Mays L.) for Grain Yield and Yield Related Traits at Kulumsa, Southeastern Ethiopia

\begin{tabular}{|c|c|c|c|c|c|c|c|c|c|c|}
\hline L20 x T2 & 25.62 & 28.80 & 13.10 & 10.64 & $28.27^{*}$ & $21.65^{*}$ & 6.67 & 4.14 & $16.25^{* *}$ & $17.72^{* *}$ \\
\hline L21 x T1 & 5.56 & 8.23 & 18.64 & 16.06 & $21.64^{*}$ & 15.36 & 7.45 & 4.91 & $11.25^{*}$ & $12.66^{* *}$ \\
\hline L21 x T2 & $62.22^{* *}$ & $66.33^{* *}$ & 19.70 & 17.09 & 16.06 & 10.06 & 2.73 & 0.30 & $9.50^{*}$ & $10.89^{*}$ \\
\hline SE $( \pm)$ & 1.23 & & 36.12 & & 2.25 & & 0.39 & & 0.27 & \\
\hline CD 0.05 & 5.02 & & 80.92 & & 6.16 & & 1.40 & & 0.34 & \\
\hline CD 0.01 & 6.64 & & 106.88 & & 8.13 & & 1.85 & & 0.46 & \\
\hline
\end{tabular}

Note: $S E(m \pm)=$ standard error of a mean, $C D=$ critical difference.

Ear length: In case of ear length, standard heterosis varied from -1.94 (L7 x T1) to $12.00 \%$ (L13 x T2) over Jibat. Although eleven hybrids showed significance positive standard heterosis. The result of standard heterosis computed relative to Wenchi showed that two crosses manifested positive and highly significant heterosis varying from -4.26 (L7 x T1) to $9.35 \%$ (L13 x T2) (Table 4). Amiruzzaman et al. (2010) reported significant positive and negative standard heterosis for ear length in maize.

\section{Discussions}

Analysis of variance showed significant difference among crosses. This indicates that crosses were sufficiently different from each other and selection is possible to identify the most desirable crosses. In line to these finding also different authors reported significant difference among crosses in yield and yield related traits in different parts of Ethiopia Legesse et al. (2009), Mosisa et al. 2011 and Shushay (2014). Percent heterosis over commercial checks was calculated for grain yield and twelve yield related traits (Table 3 and 4). Considerable amount of heterosis obtained for yield and yield related traits. Shushay (2014) and Mahantesh (2006) observed degree of heterosis for yield and its related traits in maize. Crosses showed heterosis in negative direction is considered to be desirable for days to 50 per cent tasseling, days to 50 per cent silking and days to maturity. In developing early maturing varieties while for the crosses shown positive and significant standard heterosis the reverse is true. Earliness is a desirable character as it is useful in multiple cropping and increases water land use efficiency. Negative standard heterosis is required for traits like plant height, disease and pest reaction, ear height and days to maturity. Crosses that showed negative days to anthesis, silking and days to amount of heterosis was obtained over best standard maturity stands for earliness of the crosses while for crosses shown positive standard heterosis the reverse is true. The current finding is also similar with the findings of Shushay (2014) and Mahantesh (2006). But negative heterosis for plant height and ear height is desirable for breeding short statured hybrids and which implying that these hybrids would mature earlier. Number of kernel per row and thousand kernel weights is an important yield factor and is commonly used as selection criteria in maize breeding programs and positively correlate with grain yield.

\section{CONCLUSION}

In this study, the maximum positive and significance standard heterosis $(69.63 \%)$ was observed for biomass yield, followed by number grain yield (44.54\%) and number of kernels row per ear $(43.46 \%)$ and thousand kernels weight ( $34.06 \%$ ) over the best checks Jibat. It is observed from the crosses L17 X T2, L6 XT2, L12 X T1 and L3 X T2, respectively. The existence of genetic variation for grain yield, and yield related traits give further direction for maize breeders especially those who are interested in heterosis breeding. However, further evaluation of these and other hybrids at more locations and over years is advisable to confirm the promising results observed in present study. In general, it may be concluded that the information from this study could be valuable for researchers who intend to develop high yielding varieties of maize.

\section{REFERENCES}

[1] Amiruzzaman, M., M.A. Islam, L. Hassan and M.M. Rohman, 2010. Combining ability and heterosis for yield and Component characters in maize. Academic J. Plant Sci. 3(2): 79-84.

[2] Demissew, A., S. Hussein, J. Derera, and M.Laing. 2013. Farmers' perceptions of maize roduction systems and breeding priorities, and their implications for the adoption of new varieties in selected areas of the highland agro-ecology of Ethiopia. Journal of Agricultural Science 5:159-172.

[3] Duvic, D. N., 1999. Commercial strategies for exploiting hetrosis.. In: J.G. Coors and S. pandey (eds.). The genetics and exploitation on heterosis in Crops. ASA, CSSA, SSSA Inc., adison, Wisconsin, USA. pp. 295304

International Journal of Research Studies in Agricultural Sciences (IJRSAS)

Page $\mid 6$ 
[4] Falconer DS and Mackay TFC (1996). Introduction to quantitative genetics.4 $4^{\text {th }}$ ed. Longman, London.pp.275-276.

[5] Gupta, S.C., A.K. Nagda, and G.K. Kulmi, 1994. Economic heterosis in double cross hybrids of. Crop Res. 8: 634-636.

[6] Hunter, R.B., 1999. Heterosis for the development and promotion of the seed industry.In: J.G. Coors and S. pandey (eds.). The genetics and exploitation on heterosis in crops. ASA,CSSA, SSSA Inc., Madison, Wisconsin, USA. Pp.305-311.

[7] Legesse, B.W., K.V. Pixley and A.M. Botha, 2009 Combining Ability and Heterotic Grouping of Highland Transition Maize Inbred Lines. Maydica 54: 1-9

[8] Mahantesh, 2006. Combining ability of and heterosis analysis for grain yield components in single cross hybrids of maize (Zea mays L.). M.Sc. Thesis. College of agriculture, Dharwad University of agricultural sciences. 103p.

[9] Mosisa, W., W. Legesse, T. Berhanu, D. Girma, A. Girum, A. Wende, K. Tolera, B. Gezahegn, W. Dagne, A. Solomon, Z. Habtamu, Y. Kasa, C. Temesgen, J. Habte, N. Demoz and B. Getachew, 2011. Genetic improvement of Maize for Mid- Altitude and Lowland Sub-Humid Agro- Ecologies of Ethiopia. Proceedings of the $3^{\text {rd }}$ National Maize Workshop of Ethiopia. Addis Ababa, National Maize Workshop of Ethiopia. Addis Ababa, Ethiopia: pp: 24-34.

[10] Ram, Hari Har. 2011. Crop Breeding and Biotechnology. Kalyani Publishers, Ludhiana,India.pp 733.

[11] Panse V.G. and P.V. Sukhatme, 1961. Statistical Methods for Agric. Workers, ICAR Publication New Delhi, pp: 145.

[12] Shull, G.H., 1952. Beginning of the heterosis concept. In: J.W. Gowen (ed.). Heterosis. Halfer Publishing Company, New York. Pp.14-48

[13] Shushay W (2014). Standard Heterosis of Maize (Zea mays L.) Inbred Lines for Grain Yield and Yield Related Traits in Central Rift Valley of Ethiopia. Journal of Biology, Agriculture and Healthcare, 4(23):31-37

[14] Stuber, C. W., 1994. Heterosis in Plant Breeding. Plant Breed. Rev. 12:227-251

[15] Surendran, C., M. Paramthma, and R.S. Vinayarai, 1994. Use of biometrical techniques in tree improvement. The Malaysian Forester, 57:132-139

[16] Tiwari, V.K., 2003, Studies on heterosis, combining ability, inbreeding tolerance and phenotypic stability in intra-and inter-group single cross hybrids of maize (Zea mays L.). Ph.D. Thesis.GBPUA and T, Pantnagar, India. pp. 173-197.

[17] Twumasi, A.S., Kassa, Y. and Gudeta, N., 2003.Exploitation of Combining Ability and Heterotic Responses in Maize Germplasm to Develop Cultivars for the Eastern Africa Highlands. pp.282-283.

[18] Venugopal, M., N.A. Ansani and G.K. Murthy, 2002. Heterosis and its component characters in maize (Zea mays L.). Crop Res. 3: 72-74.

[19] Virmani, S.S. and I.B. Edwards, 1983. Current status and future prospects for breeding hybrid rice and wheat. Adv. Agron. 36: 145-157.

[20] Wali, M.C., R.M. Kachapur, C.P. Chandrashekhar, V.R. Kulkarni and S.B. Devaranavadagi, 2010.Gene action and combining ability studies in single cross hybrids of maize (Zea mays L.).Karnataka, J. Agric. Sci. 23(4): 557-562.

Citation: Abiy Balcha Gebre, et.al, "Standard Heterosis of Hybrids Maize (Zea Mays L.) for Grain Yield and Yield Related Traits at Kulumsa, Southeastern Ethiopia" International Journal of Research Studies in Agricultural Sciences (IJRSAS), 2019; 5(9), pp. 1-7, http://dx.doi.org/10.20431/2454-6224.0509001

Copyright: (C) 2019 Authors. This is an open-access article distributed under the terms of the Creative Commons Attribution License, which permits unrestricted use, distribution, and reproduction in any medium, provided the original author and source are credited. 